\title{
萗酰亚胺-多胺缀合物的合成、生物活性和荧光光谱
}

\author{
田智勇 ${ }^{a}$ 苏雷朋 ${ }^{a}$ 谢松强 ${ }^{a}$ 赵 瑾 ${ }^{a, b}$ 王超杰*,a,b \\ ( ${ }^{a}$ 河南大学化学生物学研究所 开封 475004) \\ ( ${ }^{b}$ 河南大学天然药物与免疫工程重点实验室 开封 475004)
}

\begin{abstract}
摘要 合成了 9 个新的荎酰亚胺一多胺缀合物, 化合物的结构经元素分析, ${ }^{1} \mathrm{H} N M R,{ }^{13} \mathrm{C} N M R$ 和 MS 确证. 经 MTT [3-(4,5-二甲基噻唑-2)-2,5-二苯基四氮唑溴盐]法对白血病细胞(K562)、人乳腺癌细胞(MB-231)和肝癌细胞(7721)体外活 性测试. 结果显示多数化合物对肿瘤细胞具有抑作用, 尤其是化合物 $\mathbf{5 a}$ 的抗肿瘤活性优于处于 III 期临床试验阶段药 物氨荎非特(Amonafide). 化合物 $\mathbf{5} \mathbf{a}$ 与鲱鱼精 DNA-EB 作用的苂光光谱研究提示: DNA-EB 与菜酰亚胺-多胺作用引起 的荧光淬灭机制属于静态淬灭, DNA 与菜酰亚胺-多胺物结合模式是扞插结合.
\end{abstract}

关键词＼cjkstart薺酰亚胺; 多胺缀合物; 合成; 抗肿瘤活性; 荧光光谱

\section{Synthesis, Biological Activitity and Fluorescence Spectroscopy of Naphthalimide-polyamine Conjugates}

\author{
Tian, Zhiyong $^{a} \quad$ Su, Leipeng ${ }^{a} \quad$ Xie, Songqiang ${ }^{a} \quad$ Zhao, Jin $^{a, b} \quad$ Wang, Chaojie ${ }^{*, a, b}$ \\ ( ${ }^{a}$ Institute of Chemical Biology, Henan University, Kaifeng 475004) \\ ( ${ }^{b}$ Key Laboratory of Natural Medicine and Immuno-Engineering, Henan University, Kaifeng 475004)
}

\begin{abstract}
Nine novel naphthalimide-polyamine conjugates were synthesized and their structures were confirmed by elemental analysis, ${ }^{1} \mathrm{H}$ NMR, ${ }^{13} \mathrm{C}$ NMR and MS techniques. Their antitumor activities in vitro using MTT [3-(4,5-dimethylthiazol2-yl)-2,5-diphenyltetrazolium bromide] assay were assessed on leukemia cells (K562), human breast cancer cells (MB-231) and human hepatoma cells (7721). The results showed that most of them exhibited antitumor activities. Especially, compound 5a was better than amonafide which has been in phase III clinical trials. Fluorescence data revealed that the fluorescence quenching of herring sperm DNA-EB complex by compound 5a was a static quenching type and naphthalimide-polyamine conjugates intercalate into the DNA base pairs.
\end{abstract}

Keywords naphthalimide; polyamine conjugates; synthesis; antitumor activity; fluorescence spectroscopy

萗酰亚胺作为 DNA 嵌入剂, 具有良好的抗肿瘤活 性, 作为代表性䓫酰亚胺类化合物氨慕非特 (Amonafide)已经处入 III 期临床试验阶段 ${ }^{[1 \sim 3]}$. 但由于水 溶性较差, 影响了它在临床中的进一步研究应用. 因此 对菜酰亚胺进行结构修饰以开发活性好、毒副作用小、 水溶性更好的芸酰亚胺类抗肿瘤药物具有深远的意义.

多胺包括精胺、亚精胺、腐胺是天然的有机胺类化 合物，具有广泛的生理活性. 随着国际上对各类多胺的 合成、代谢机制、结构与生理活性关系等方面进行的多
学科交叉研究, 特别是 1995 年报道了用多胺作为载体 搭载抗癌药物分子以来, 多胺的靶向给药能力逐渐引起 了人们的注意, 多胺类化合物的合成及其构效关系的研 究成为了当前国际上新药研究的主流之一. 因此利用多 胺作载体运载抗癌药物分子既可增强药物抗肿瘤活性, 又能够达到药物作用的靶向性 ${ }^{[4-8]}$.

DNA 是人类生命重要的遗传物质，同时也是许多 药物作用的靶点. DNA 嵌入剂是通过其本身平面结构插 入到 DNA 螺旋的相邻两个碱基对之间发挥作用的.

\footnotetext{
*E-mail: wcjsxq@henu.edu.cn

Received January 4, 2013; revised January 31, 2013; published online February 5, 2013.

Project supported by the China Postdoctoral Science Foundation Funded Project (No. 20110490991), the Natural Science Foundation of Henan Province (No. 112300410181), the Henan Provincial Programs for Science and Technology Development (No. 132102310026) and the Natural Science Foundation of Education Department of Henan Province (No. 2011B3500001).

中国博士后科学基金(No. 20110490991)、河南省自然科学(No. 112300410181)、河南省科技攻关(No. 132102310026)和河南省教育厅自然科学基金(No. 2011B3500001)资助项目.
} 
我们前期工作发现菜酰亚胺-多胺缀合物具有良好 的抗肿瘤活性、对 DNA 有嵌入作用, 并能够引起肿瘤 细胞调亡 ${ }^{[9 \sim 13]}$, 但并没有对 DNA(包括 DNA-EB 复合物) 与荟酰亚胺-多胺作用引起的荧光淬灭机制及作用模式 进行深入研究. 同时在文献[14]调研中也发现 3,6-二硝 基-荟酰亚胺衍生物有着较强的体内外抑制白血病和黑 素瘤的活性. 因此在此基础上我们又合成了包括 6 个 3,6-二硝基-䒬酰亚胺-三胺缀合物(Scheme 1)和 3 个荎 酰亚胺-二胺缀合物在内的 9 个新的菜酰亚胺-多胺缀合 物(Scheme 2), 并进行了生物活性评价和苂光光谱学研 究.
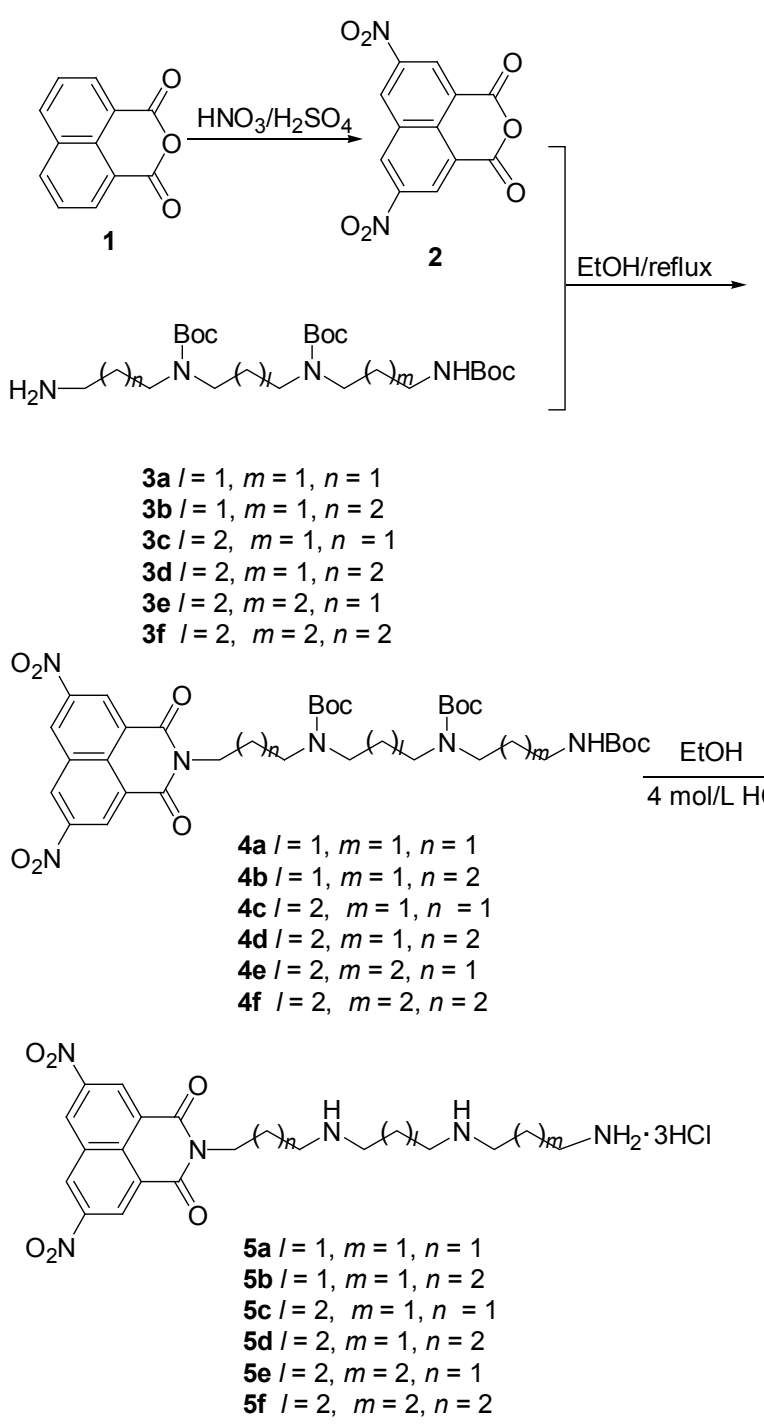

Scheme 1

\section{1 结果与讨论}

\section{1 目标化合物结构确认}

目标化合物的 ${ }^{1} \mathrm{H}$ NMR 中, 与文献[9]相比, 化合物 $5 \mathbf{a} \sim 5 \mathbf{f}$ 的芳环由于双硝基的引入, 致使芳环的信号峰向

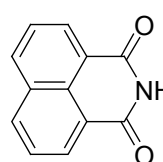

6

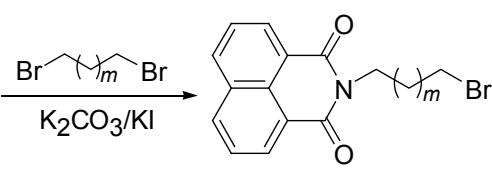

7

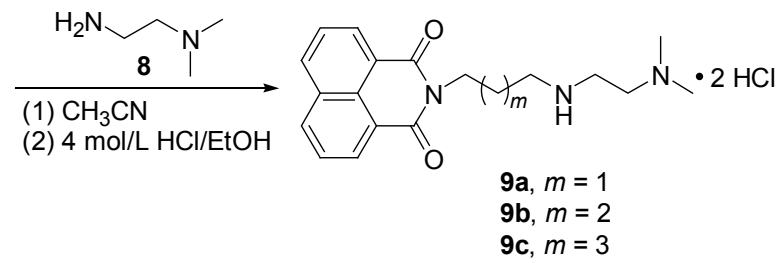

Scheme 2
低场移动 $\delta \quad 0.8 \sim 1.3$. 而化合物 $9 \mathbf{a} \sim 9 \mathbf{c}$ 无硝基引入, 所 以芳环的信号峰和文献[9]一样，一般出现在 $\delta 7.0 \sim 8.0$ 之间; 化合物 $5 \mathbf{a} \sim \mathbf{5 f}$ 的侧链与酰亚胺氮原子相连亚甲 基的信号峰一般是出现在 $\delta 4.0$ 左右的三重峰; 多胺骨 架上与氮原子相连的 5 个亚甲基的信号峰一般出现在 $\delta$ 3.05 3.35 之间, 为多重峰, 其余的亚甲基的信号峰一 般出现在 $\delta 1.70 \sim 2.26$ 之间, 为多重峰, 化合物不同, 则 亚甲基数目不同. 化合物 $9 a \sim 9 c$ 的侧链除 $9 a$ 的 $\mathrm{CONH}_{2}$ 与 $\mathrm{NCH}_{2}$ 信号峰重叠形成多重峰外, 化合物 $9 \mathbf{b} \sim 9 \mathbf{c}$ 侧链 与酰亚胺氮原子相连亚甲基的信号峰一般是出现在 $\delta$ 3.80 左右的三重峰, 在 $\delta 3.50 \sim 3.70$ 之间有两个 $\mathrm{NCH}_{2}$ 的多重峰, 有趣的是化合物 $9 a \sim 9 \mathrm{c}$ 在 $\delta 3.0$ 左右有 1 个 $\mathrm{NCH}_{2}$ 的三重峰, 更重要的是, 化合物 $9 \mathrm{a} \sim 9 \mathrm{c}$ 在 $\delta 3.0$ 左 右有两个甲基单峰.

目标化合物的 ${ }^{13} \mathrm{C}$ NMR 中, 羰基信号出现在 $\delta 165$ 左右. 化合物 9a $\sim 9 \mathbf{c}$ 的芳环信号峰一般出现在 $\delta 118 \sim$ 136 之间并表现出结构对称性, 但与文献[9]相比, 化合 物 $\mathbf{5 a} \sim \mathbf{5 f}$ 由于硝基引入致使与硝基相连的芳环碳原子 向低场移动约 $\delta 15$. 化合物 $\mathbf{5 a} \sim \mathbf{5} \mathbf{f}$ 与酰亚胺氮原子相连 的亚甲基的信号峰一般出现在 $\delta 47$ 左右，接下来是多胺 骨架上与其他氮原子相连的 5 个亚甲基的信号峰，一般 出现在 $\delta 47 \sim 35$ 之间; 与氮原子不相连的亚甲基的信号 峰一般出现在 $\delta 22 \sim 25$ 之间较窄的范围内; 化合物 5a $\sim 5$ 的区别则是表现在亚甲基数目上的差异. 化合物 $9 \mathrm{a} \sim 9 \mathrm{c}$ 侧链中与酰亚胺氮原子相连的亚甲基的信号峰 一般出现在 $\delta 50 \sim 55$, 接下来是多胺骨架上与氮原子相 连的 3 个亚甲基的信号峰和 2 个甲基的信号峰, 其中两 个甲基的信号峰在 $\delta 43$ 左右, 剩下的亚甲基是与氮原子 不相连的, 它们的信号峰一般出现在 $\delta 20 \sim 20$ 之间较窄 的范围内; 化合物 9a $\sim 9 \mathrm{c}$ 的区别和化合物 $5 \mathrm{a} \sim 5 \mathrm{f}$ 的区 别一样, 表现出亚甲基数目上的差异.

元素分析的结果显示: 目标化合物中均含有一定量 的水, 这与盐酸盐有较强的吸水性能有关. 但经加水修 正后, 元素含量均在误差范围内 $(0.4 \%)$. 
质谱分子离子峰表现为 $\mathrm{M}+\mathrm{H}-n \mathrm{HCl}(n=2$ 或 3).

根据以上分析, 可以证明所合成的 9 个目标化合物 结构与预期结果相符.

\section{2 体外抗肿瘤活性}

所有目标化合物, 采用 MTT 法 ${ }^{[15]}$ 进行活性初步篎 选, 对照品为处于 III 期临床试验阶段药物氨荎非特 (Amonafide), 结果见表 1. 表中所列数据为化合物浓度 分别为 $10 \times 10^{-6}, 30 \times 10^{-6} \mathrm{~mol} \cdot \mathrm{L}^{-1}$ 时的对肿瘤细胞抑 制率，抑制率越高，表示化合物的细胞毒性越强.

表 1 化合物 5a $\sim 5 f$ 和 9a $\sim 9$ 的体外生物活性

Table 1 Biological activities of compounds 5a $\sim 5 \mathbf{f}$ and $9 a \sim 9 f$

\begin{tabular}{|c|c|c|c|c|c|c|}
\hline \multirow{3}{*}{ Compd. } & \multicolumn{6}{|c|}{ Inhibition rate/\% } \\
\hline & \multicolumn{2}{|c|}{7721} & \multicolumn{2}{|c|}{ MB231 } & \multicolumn{2}{|c|}{ K562 } \\
\hline & $\mathrm{A}^{a}$ & $\mathrm{~B}^{b}$ & $\mathrm{~A}^{a}$ & $\mathrm{~B}^{b}$ & $\mathrm{~A}^{a}$ & $\mathrm{~B}^{b}$ \\
\hline $5 \mathbf{5}$ & 38.2 & 65.4 & 46.9 & 51.0 & 45.8 & 69.4 \\
\hline $5 \mathbf{b}$ & 17.6 & 30.8 & 42.3 & 50.4 & 32.6 & 56.5 \\
\hline $5 c$ & 20.6 & 36.5 & 41.7 & 44.7 & 33.9 & 36.1 \\
\hline 5d & 0 & 0 & 44.3 & 44.6 & 31.9 & 33.5 \\
\hline $5 e$ & 0 & 0 & 42.6 & 50.3 & 31.3 & 50.3 \\
\hline $5 f$ & 32.5 & 61.3 & 40.3 & 45.6 & 28.8 & 31.3 \\
\hline $9 a$ & 0 & 0 & 4.90 & 28.6 & 28.8 & 43.5 \\
\hline $9 b$ & 0 & 0 & 2.80 & 34.4 & 29.5 & 44.5 \\
\hline $9 \mathrm{c}$ & 0 & 0 & 0.05 & 33.6 & 35.3 & 49.3 \\
\hline Amonafide & 20.3 & 59.6 & 12.3 & 20.4 & 17.5 & 45.2 \\
\hline
\end{tabular}

${ }^{a} \mathrm{~A}: c=10 \times 10^{-6} \mathrm{~mol} \cdot \mathrm{L}^{-1},{ }^{b} \mathrm{~B}: c=30 \times 10^{-6} \mathrm{~mol} \cdot \mathrm{L}^{-1}$.

由表 1 可以看出, 氨萘非特对 3 种癌细胞具有很强 的选择性, 对人乳腺癌细胞(MB-231)活性较差, 而对白 血病细胞(K562)和肝癌细胞(7721)活性较好. 目标化合 物也显示出对被测肿瘤细胞的抗肿瘤活性或选择性. 如 化合物 5a 对所有被测肿瘤细胞具有较好的抗肿瘤活性, 优于处于 III 期临床试验阶段的对照药物 Amonafide; 5b 和 5e 对 7721 活性较差, 但对 K562 和 MB-231 具有一定 抗肿瘤活性; 化合物 5c 和 5d 对 MB-231 有一定活性, 但 对 K562 和 7721 活性较差; 化合物 $5 \mathbf{f}$ 对 7721 和 MB-231 活性较好, 但对 K562 活性较差. 化合物 9a 9c 仅对 K562 有抑制作用，但对 MB-231 抑制作用较差，对 7721 完全没有活性.

\section{3 荧光光谱研究}

\subsection{1 荧光淬灭方式的判断}

为了深入考察目标化合物与 DNA 作用情况, 选用 化合物 5a 与鲱鱼精 DNA 作用. 但由于硝基具有强的吸 电子作用, 导致 3,6-二硝基-䒬酰亚胺-三胺缀合物本身 荧光消失, 从而无法观察 DNA 与 3,6-二硝基-菜酰亚 三胺缀合物作用的苂光淬灭现象 ${ }^{[16]}$. 因此利用 DNA 与 溴化乙锭(EB)形成复合物, 其混合溶液在 $600 \mathrm{~nm}$ 左右 处出现明显的苂光, 当结合能力比 $\mathrm{EB}$ 更强的嵌入剂加 入后, 会与体系中的 $\mathrm{EB}$ 争夺与 $\mathrm{DNA}$ 上的结合点位置,
使体系中 $\mathrm{EB}$ 被置换出来，从而产生荧光瘁灭现象的性 质，进行化合物 $\mathbf{5 a}$ 与 DNA-EB 复合物作用，结果见图 1.

从图 1 中可以看出, 当化合物 $\mathbf{5 a}$ 加入混合溶液后, 引起荧光淬灭. 苂光瘁灭作用因瘁灭机制不同而分为动 态淬灭和静态淬灭. 判断化合物 5a 与 DNA-EB 作用引 起苂光淬灭机制, 对药物与 DNA 结合模式至关重要.

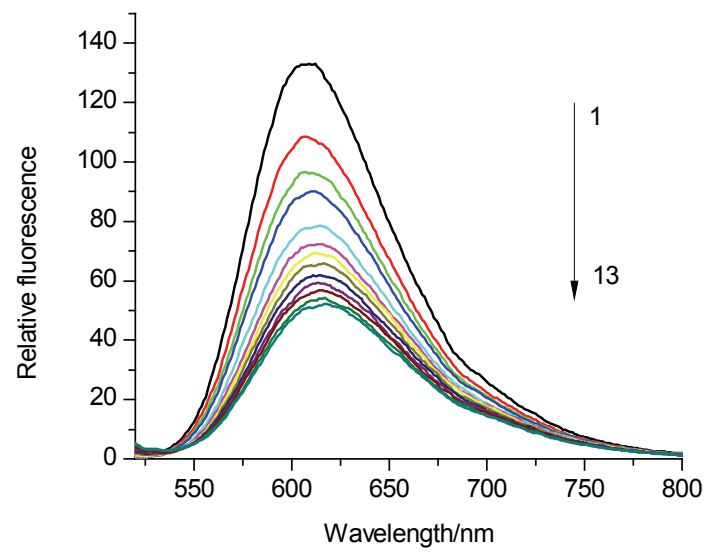

图 1 化合物 5a 与鲱鱼精 DNA-EB 作用苂光图

Figure 1 Fluorescence spectroscopy of compound 5a with herring sperm DNA-EB

Numbers $1 \sim 13$ indicated the 5 a concentration of $0.0,4 \times 10^{-6}, 8 \times 10^{-6}$, $12 \times 10^{-6}, 24 \times 10^{-6}, 36 \times 10^{-6}, 48 \times 10^{-6}, 60 \times 10^{-6}, 72 \times 10^{-6}, 84 \times$ $10^{-6}, 96 \times 10^{-6}, 108 \times 10^{-6}$ and $120 \times 10^{-6} \mathrm{~mol} \cdot \mathrm{L}^{-1}$, respectively. DNA and $\mathrm{EB}$ applied was $13.7 \times 10^{-6}$ and $15.7 \times 10^{-6} \mathrm{~mol} \cdot \mathrm{L}^{-1}$, respectively. Scan condition: $\mathrm{EX}=510 \mathrm{~nm}, \mathrm{EM}=520 \sim 800 \mathrm{~nm}$; Slits of both $\mathrm{EX}$ and EM were $5 \mathrm{~nm}$.

化合物与 DNA-EB 复合物作用的动态淬灭过程反 映了化合物 $\mathbf{5 a}$ 与 DNA-EB 复合物的激发态分子之间碰 撞所发生的相互作用过程. 这个过程是用 Stern-Volmer 方程来进行描述的, 即:

$$
F_{0} / F=1+K_{\mathrm{SV}} c_{\mathbf{5 a}}
$$

其中 $F_{0}$ 和 $F$ 分别为 EB-DNA 和化合物 $5 \mathbf{a}$ 作用前后的荧 光强度, $K_{\mathrm{SV}}$ 为动态淬灭常数, 用来衡量化合物 $\mathbf{5 a}$ 的淬 灭速率, $c_{\mathbf{5}}$ 表示化合物 $\mathbf{5 a}$ 的浓度. 以 $F_{0} / F$ 对 $c_{\mathbf{5}}$ 作图, 可 以得到药物的 Stern-Volmer 猝灭曲线 ${ }^{[17,18]}$, 结果见图 2 . 化合物 5a 与 DNA-EB 复合物作用的静态淬灭过程反映 了化合物 $\mathbf{5}$ 与 DNA-EB 复合物子之间借助分子间力, 彼此结合形成了具有一定结构的分子复合物, 而导致荧 光体荧光减弱的现象，这个过程是用 Lineweaver-Burk 双倒数方程来进行描述的, 方程式如下:

$$
\left(I_{\mathrm{F}}^{0}-I_{\mathrm{F}}\right)^{-1}=I_{\mathrm{F}}^{0}+K_{\mathrm{LB}}^{-1}\left(I_{\mathrm{F}}^{0}\right)^{-1} c_{\mathrm{q}}^{-1}
$$

其中， $I_{\mathrm{F}}^{0}$ 和 $I_{\mathrm{F}}$ 分别为化合物 $\mathbf{5 a}$ 加入 DNA-EB 复合物前 后的荧光强度, $K_{\mathrm{LB}}$ 为静态荧光淬灭过程中复合物的形 成常数, 它反映了在静态淬灭过程中 DNA-EB复合物与 
化合物 5a 的结合反应达到平衡时的量效关系. 以 $\left(I_{\mathrm{F}}^{0}-\right.$ $\left.I_{\mathrm{F}}\right)^{-1}$ 对 $\left(I_{\mathrm{F}}^{0}\right)^{-1} c_{\mathrm{q}}^{-1}$ 作图, 就得到 Lineweaver-Burk 双倒数 曲线 ${ }^{[17]}$.

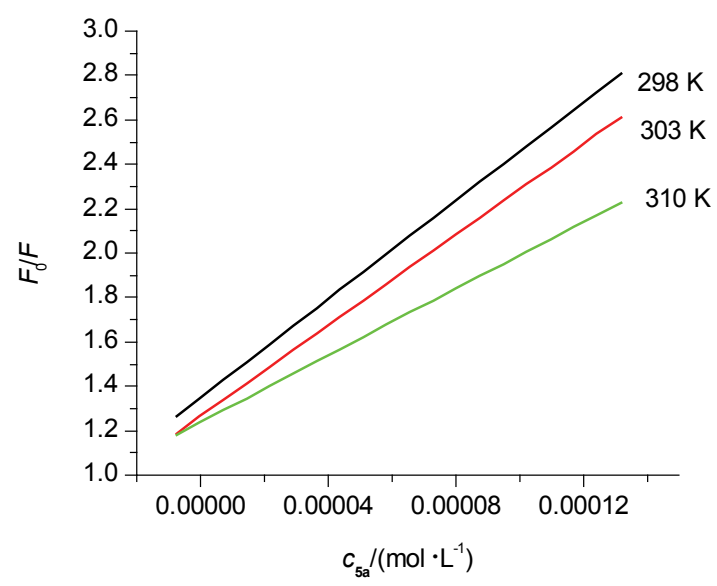

图 2 化合物 5a 与鲱鱼精 DNA-EB 在不同温度下作用的 Stern-Volmer 猝灭曲线线性拟合图

Figure 2 Stern-Volmer fit linear plot of fluorescence quenching of herring sperm DNA-EB by compound $\mathbf{5 a}$ at different temperatures

根据分别在 $25,30,37{ }^{\circ} \mathrm{C}$ 时获得的化合物 $\mathbf{5 a}$ 与 DNA-EB 复合物作用的荧光光谱数据, 分别做出 3 个不 同温度下的 Stern-Volmer 曲线和 Lineweaver-Burk 双倒 数曲线, 然后对实验数据进行线性拟合, 即得 $K_{\mathrm{SV}}$ 和 $K_{\mathrm{LB}}$ 的值, 结果见表 2 .

表 2 化合物 $5 \mathbf{a}$ 与鲱鱼精 DNA 作用淬灭常数

Table 2 Quenching constants of the interaction between compound $\mathbf{5 a}$ and herring sperm DNA

\begin{tabular}{cccc}
\hline$T / \mathrm{K}$ & $K_{\mathrm{SV}} /\left({\left.\mathrm{L} \cdot \mathrm{mol}^{-1}\right)}^{-1}\right.$ & $K_{\mathrm{q}} /\left(\mathrm{L} \cdot \mathrm{mol}^{-1}\right)$ & $K_{\mathrm{LB}} /\left({\left.\mathrm{L} \cdot \mathrm{mol}^{-1}\right)}^{12}\right.$ \\
\hline 298 & $1.106 \times 10^{4}$ & $1.106 \times 10^{12}$ & $6.670 \times 10^{4}$ \\
303 & $1.020 \times 10^{4}$ & $1.076 \times 10^{12}$ & $5.090 \times 10^{4}$ \\
310 & $7.502 \times 10^{3}$ & $7.502 \times 10^{11}$ & $3.598 \times 10^{4}$ \\
\hline
\end{tabular}

一般来讲, 动态淬灭作用的 $K_{\mathrm{q}}$ 值一般都小于 $2.000 \times 10^{10} \mathrm{~L} \cdot \mathrm{mol}^{-1[19]}$, 但表 2 中的 $K_{\mathrm{q}}$ 值远大于

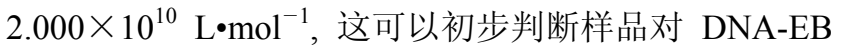
的淬灭方式为静态淬灭. 据文献[20]报道温度升高将增 大分子扩散系数, 从而增大发生碰撞淬灭的可能性, 如 果是动态淬灭, 则 $K_{\mathrm{SV}}$ 应随温度的升高而增大, 同时温 度升高将导致配合物的稳定性降低, 从而减小形成配合
物的可能性; 如果是静态淬灭, 则 $K_{\mathrm{LB}}$ 随着温度的升高 而应该减小. 表 2 中的 $K_{\mathrm{SV}}$ 随温度的升高到而减小, 则 表明样品对 DNA-EB 的淬灭方式不是动态淬灭; $K_{\mathrm{LB}}$ 随 着温度升高的而减小, 这些结果进一步表明样品对 DNA-EB 的荧光淬灭方式为静态淬灭, 初步说明了样品 与 DNA 结合是扞插结合 ${ }^{[17]}$.

\subsection{2 化合物与 DNA 结合方式判定}

由于静态猝灭过程, 苂光强度与淬灭剂的关系符合 以下方程: $\log \left[\left(F_{0}-F\right) / F\right]=\log K+n \log [Q]$, 其中 $F_{0}$ 和 $F$ 分别是化合物加入 DNA-EB 体系前后的荧光强度, $K$ 为 化合物 $\mathbf{5 a}$ 与 DNA 的结合常数, $n$ 为结合位点数, $[Q]$ 是化 合物 $5 \mathbf{a}$ 的总的浓度. 所以根据此方程, 以 $\log \left[\left(F_{0}-\right.\right.$ $F) / F]$ 对 $\log [Q]$ 作图, 然后对实验数据进行线性拟合, 由 直线的截距和斜率可求得化合物 $\mathbf{5 a}$ 与 DNA 分子的在不 同温度下的结合常数 $K$ 和结合位点数 $n$, 结果见表 3 和 图 3.

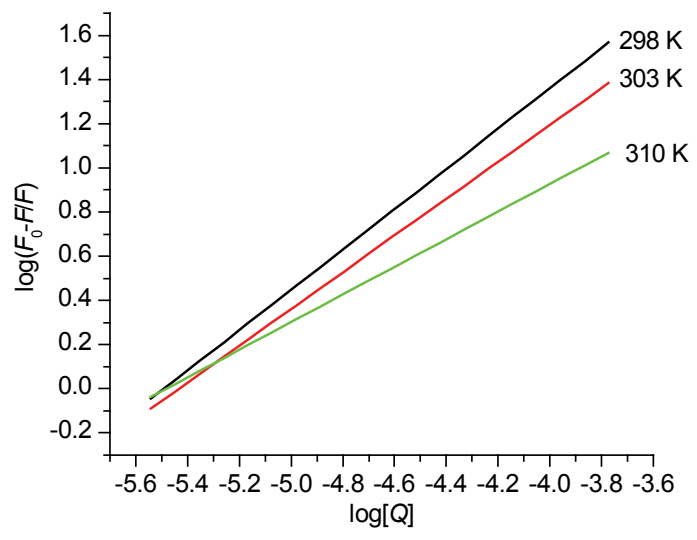

图 3 不同温度下的 $\log \left[\left(F_{0}-F\right) / F\right]$ 对 $\log [Q]$ 线性拟合图 Figure 3 Fit linear plot of $\log \left[\left(F_{0}-F\right) / \mathrm{F}\right]$ vs $\log [Q]$ at different temperatures

利用以下公式计算化合物 5a 与 DNA-EB 复合物作 用的热力学函数 ${ }^{[18,21 ~ 23]}$, 焓变 $\Delta H$ 熵变 $\Delta S$ 和吉布斯自由 能变 $\Delta G$ 的数值, 即:

$$
\begin{aligned}
& \ln \left(K_{2} / K_{1}\right)=\left(1 / T_{1}-1 / T_{2}\right) \Delta H / R \\
& \Delta G=-R T \ln K=\Delta H-T \Delta S
\end{aligned}
$$

其中, $R$ 为气体常数, $T$ 为温度, $K$ 为结合常数. 当温度变 化不大时, 焓变 $\Delta H$ 可以看作是一个常数, 结果见表 3 .

表 3 中数据 $\Delta H<0$, 则表明样品与 DNA 的结合是放

表 3 化合物 $5 \mathbf{a}$ 与鲱鱼精 DNA 在不同温度下作用的结合常数及热力学参数

Table 3 Binding constants and thermodynamic parameters of the interaction between compound $\mathbf{5 a}$ and herring sperm DNA at different

\begin{tabular}{|c|c|c|c|c|c|c|}
\hline$T / \mathrm{K}$ & $K /\left(\mathrm{kJ} \bullet \mathrm{mol}^{-1}\right)$ & $n$ & $\Delta G /\left(\mathrm{kJ} \bullet \mathrm{mol}^{-1}\right)$ & $\Delta H /\left(\mathrm{kJ} \bullet \mathrm{mol}^{-1}\right)$ & $\Delta S /\left(\mathrm{kJ} \bullet \mathrm{mol}^{-1}\right)$ & $R^{a}$ \\
\hline 298 & $1.019 \times 10^{5}$ & 0.91 & -28.571 & -166.845 & -0.464 & 0.9725 \\
\hline 303 & $3.354 \times 10^{4}$ & 0.83 & -26.251 & -166.845 & -0.464 & 0.9669 \\
\hline 310 & $2.686 \times 10^{3}$ & 0.63 & -20.351 & -166.845 & -0.464 & 0.9755 \\
\hline
\end{tabular}
temperatures

${ }^{a}$ Correlation cocefficient. 
的. 由 Ross 等 ${ }^{[17,18,21]}$ 总结出的判断生物大分子与小分子 结合力类型的热力学规律可以用来判断结合力类型: 疏 水作用力其热力学特征为 $\Delta H>0$ 和 $\Delta S>0$; 氢键或者范 德华力其热力学特征为 $\Delta H<0$ 和 $\Delta S<0$; 静电作用力其 热力学特征为 $\Delta H<0$. 结合表 3 中数据均小于 0 , 由此可 判断样品与 DNA 的结合是焓熵协同驱动过程, 而且它 们之间的结合力类型以氢键和静电作用力为主. Takenaka 等 ${ }^{[24]}$ 研究发现, 氢键作用是药物与 DNA 发生扦插 结合的显著特征, 所以这更进一步证明样品与 DNA 的 结合方式是扦插.

由此可推断, 样品与 DNA 相互作用的过程是放热 的焓熵协同驱动过程, 当样品与 DNA 相互作用时, 样 品的平面荎环结构插入 DNA 的双螺旋结构的碱基对之 间，且相互作用力类型为氢键和静电作用.

\section{2 结论}

合成了 9 个菜酰亚胺衍生物, 并经过 ${ }^{1} \mathrm{H} \mathrm{NMR},{ }^{13} \mathrm{C}$ NMR, MS，元素分析等结构确认.

体外生物活性显示: 所合成的多数化合物对被测肿 瘤细胞具有抑制作用. 尤其是化合物 $\mathbf{5 a}$, 抗肿瘤活性优 于处于 III 期临床试验阶段药物氨慕非特(Amonafide).

DNA-EB 与菜酰亚胺衍生物 $\mathbf{5 a}$ 作用能引起的荧光 淬灭, 通过比较在三种不同温度下的化合物 $5 \mathrm{a}$ 与鲱鱼 精 DNA-EB 相互作用的动态淬灭常数与静态淬灭常数, 判定化合物 5a 与鲱鱼精 DNA-EB 作用的苂光淬灭机制 为静态淬灭. 通过比较在三种不同温度下的化合物 $\mathbf{5 a}$ 与鲱鱼精 DNA-EB 相互作用的荧光光谱数据, 计算出了 不同温度下的结合常数, 结合位点及热力学参数. 通过 分析不同温度下的热力学参数, 判定化合物 $5 \mathbf{a}$ 与鲱鱼 精 DNA 的结合方式是扦插结合, 也就是说样品的平面 菜环结构插入 DNA 的双螺旋结构的碱基对之间, 且相 互作用力类型为氢键和静电作用, 此过程是放热的焓摘 协同驱动过程.

\section{3 实验部分}

\section{1 仪器与试剂}

Bruker AV-400 型核磁共振仪(美国); Esquire 3000 型 LC-MS 质谱仪(德国); Vario EL III 型元素分析仪(德 国); Unicam UV 500 紫外分光光度计; F-7000 FL Spectrophotometer(日本); X-6 精密显微熔点测定仪.

1,8-䒬二甲酸酐、1,8-萘二甲酰亚胺(鞍山惠丰化工 股份有限公司); 氨荟非特(本实验室合成, 经 ${ }^{1} \mathrm{H}$ NMR 结构确认); 硅胶(300 400 目, 青岛海洋硅胶干燥厂), 其他试剂均为市售分析纯，未作进一步处理.

\section{2 目标化合物合成与结构表征}

\section{$32.13,6$-二硝基-1,8-䓠二甲酸酐的合成}

在 $250 \mathrm{~mL}$ 三口烧瓶中加入 $100 \mathrm{mmol}(19.80 \mathrm{~g})$ 的 1,8-菜二甲酸酐(1)和 $100 \mathrm{~mL}$ 浓硫酸 $(98 \%)$, 搅拌使其溶 解, $0{ }^{\circ} \mathrm{C}$ 缓慢滴加由 $11.4 \mathrm{~mL}$ 浓硝酸 $65 \%$ ～ $68 \%$ 与 $50 \mathrm{~mL}$ 浓硫酸(98\%)配制而成的混酸, 滴毕 $0{ }^{\circ} \mathrm{C}$ 反应 $2.5 \mathrm{~h}$, 然 后升温至 $50{ }^{\circ} \mathrm{C}$ 继续反应 $1 \mathrm{~h}$. 反应结束后, 冷却至室温, 置于碎冰上, 抽滤, 水洗至中性, 干燥, 冰醋酸重结晶, 烘干，得淡黄色固体 $\mathbf{2}^{[14]}$, 产率 93.5\%. m.p. 204.6 $206.1{ }^{\circ} \mathrm{C} ;{ }^{1} \mathrm{H}$ NMR $\left(\mathrm{CDCl}_{3}, 400 \mathrm{MHz}\right) \delta: 9.81$ (d, $J=2.0$ $\mathrm{Hz}, 2 \mathrm{H}, \mathrm{ArH}), 9.08$ (d, $J=2.0 \mathrm{~Hz}, 2 \mathrm{H}, \mathrm{ArH})$.

\subsection{2 多胺侧链合成}

被保护的四胺 3 按参考文献[25]进行合成.

\subsubsection{3, 6-二硝基-菜酰亚胺-三胺缀合物 5 的合成}

被保护的四胺 $31 \mathrm{mmol}$ 与 3,6-二硝基-1,8 菜二甲酸 酎 $0.9 \mathrm{mmol}$ 在无水乙醇中回流 $3.5 \mathrm{~h}$, 回收乙醇进行柱 层析，得淡黄色油状物 4 .

4a: 产率 $41.67 \% .{ }^{1} \mathrm{H}$ NMR $\left(\mathrm{CDCl}_{3}, 400 \mathrm{MHz}\right) \delta$ : $9.44(\mathrm{~d}, J=2.0 \mathrm{~Hz}, 2 \mathrm{H}, \operatorname{ArH}), 9.34$ (d, $J=2.0 \mathrm{~Hz}, 2 \mathrm{H}$, ArH), 5.24 (br s, 1H, NHCO), 4.23 (t, $J=7.2 \mathrm{~Hz}, 2 \mathrm{H}$, $\left.\mathrm{CONCH}_{2}\right), 3.08 \sim 3.34\left(\mathrm{~m}, 10 \mathrm{H}, \mathrm{NCH}_{2}\right), 1.61 \sim 2.17(\mathrm{~m}$, $\left.6 \mathrm{H}, \mathrm{CH}_{2}\right), 1.43$ (s, 27H, Boc-H); ESI-MS m/z: $781.3(\mathrm{M}+$ $\mathrm{Na})^{+}$.

4b: 产率 46.04\%. ${ }^{1} \mathrm{H} \mathrm{NMR}\left(\mathrm{CDCl}_{3}, 400 \mathrm{MHz}\right) \delta$ : $9.44(\mathrm{~d}, J=2.0 \mathrm{~Hz}, 2 \mathrm{H}, \mathrm{ArH}), 9.33$ (d, $J=2.0 \mathrm{~Hz}, 2 \mathrm{H}$, ArH), 5.29 (br s, 1H, NHCO), 4.24 (t, $J=7.2 \mathrm{~Hz}, 2 \mathrm{H}$, $\left.\mathrm{CONCH}_{2}\right), 3.08 \sim 3.25\left(\mathrm{~m}, 10 \mathrm{H}, \mathrm{NCH}_{2}\right), 1.42 \sim 1.77(\mathrm{~m}$, $35 \mathrm{H}, \mathrm{CH}_{2}$, Boc-H); ESI-MS m/z: $795.4(\mathrm{M}+\mathrm{Na})^{+}$.

4c: 产率 $46.11 \% .{ }^{1} \mathrm{H} \mathrm{NMR}\left(\mathrm{CDCl}_{3}, 400 \mathrm{MHz}\right) \delta$ : $9.45(\mathrm{~d}, J=2.0 \mathrm{~Hz}, 2 \mathrm{H}, \operatorname{ArH}), 9.34(\mathrm{~d}, J=2.0 \mathrm{~Hz}, 2 \mathrm{H}$, ArH), 5.25 (br s, 1H, NHCO), 4.22 (t, $J=6.8 \mathrm{~Hz}, 2 \mathrm{H}$, $\left.\mathrm{CONCH}_{2}\right), 3.09 \sim 3.33\left(\mathrm{~m}, 10 \mathrm{H}, \mathrm{NCH}_{2}\right), 1.42 \sim 1.64(\mathrm{~m}$, $35 \mathrm{H}, \mathrm{CH}_{2}$, Boc-H); ESI-MS m/z: $795.8(\mathrm{M}+\mathrm{Na})^{+}$.

4d: 产率 $66.47 \%$. ${ }^{1} \mathrm{H} \mathrm{NMR}\left(\mathrm{CDCl}_{3}, 400 \mathrm{MHz}\right) \delta$ : 9.44 (d, $J=2.0 \mathrm{~Hz}, 2 \mathrm{H}, \operatorname{ArH}), 9.33$ (d, $J=2.0 \mathrm{~Hz}, 2 \mathrm{H}$, ArH), 5.28 (br s, 1H, NHCO), 4.24 (t, $J=6.8 \mathrm{~Hz}, 2 \mathrm{H}$, $\left.\mathrm{CONCH}_{2}\right), 3.08 \sim 3.22\left(\mathrm{~m}, 10 \mathrm{H}, \mathrm{NCH}_{2}\right), 1.42 \sim 1.74(\mathrm{~m}$, $37 \mathrm{H}, \mathrm{CH}_{2}$, Boc-H); ESI-MS m/z: $809.3(\mathrm{M}+\mathrm{Na})^{+}$.

4e: 产率 $46.04 \% .{ }^{1} \mathrm{H} \mathrm{NMR}\left(\mathrm{CDCl}_{3}, 400 \mathrm{MHz}\right) \delta$ : 9.46 (d, $J=1.6 \mathrm{~Hz}, 2 \mathrm{H}, \operatorname{ArH}), 9.33$ (d, $J=1.6 \mathrm{~Hz}, 2 \mathrm{H}$, ArH), 4.58 (brs, 1H, NHCO), 4.24 (t, $J=8.0 \mathrm{~Hz}, 2 \mathrm{H}$, $\left.\mathrm{CONCH}_{2}\right), 3.10 \sim 3.33\left(\mathrm{~m}, 10 \mathrm{H}, \mathrm{NCH}_{2}\right), 1.34 \sim 1.94(\mathrm{~m}$, $37 \mathrm{H}, \mathrm{CH}_{2}$, Boc-H); ESI-MS $m / z: 809.5(\mathrm{M}+\mathrm{Na})^{+}$.

4f: 产率 $55.56 \% .{ }^{1} \mathrm{H} \mathrm{NMR}\left(\mathrm{CDCl}_{3}, 400 \mathrm{MHz}\right) \delta: 9.45$ (d, $J=2.0 \mathrm{~Hz}, 2 \mathrm{H}, \mathrm{ArH}), 8.34$ (d, $J=2.0 \mathrm{~Hz}, 2 \mathrm{H}, \mathrm{ArH})$, 
$4.61($ br s, $1 \mathrm{H}, \mathrm{NHCO}), 4.24\left(\mathrm{t}, J=7.2 \mathrm{~Hz}, 2 \mathrm{H}, \mathrm{CONCH}_{2}\right.$ ), $3.10 \sim 3.22\left(\mathrm{~m}, 10 \mathrm{H}, \mathrm{NCH}_{2}\right), 1.42 \sim 1.71\left(\mathrm{~m}, 39 \mathrm{H}, \mathrm{CH}_{2}\right.$, Boc-H); ESI-MS $m / z$ : $823.6(\mathrm{M}+\mathrm{Na})^{+}$.

上述油状物 4 溶于适量乙醇中, 冷却至 $0{ }^{\circ} \mathrm{C}$, 分别 滴加适量的 $4 \mathrm{~mol} / \mathrm{L}$ 盐酸的乙醇溶液, 自然升至室温, 搅拌过夜至有大量固体生成, 过滤, 用重蒸无水乙醇洗 涤 3 次, 干燥得灰白色固体化合物 $\mathbf{5}^{[11]}$.

3,6-二硝基- 2-\{3-[3-(3-氨丙氨基)丙氨基]丙基 $\}-1 H$ 苯并异喹啉- 1,3(2H)-二酮三盐酸盐(5a): 产率 $53.99 \%$. m.p. $240.6 \sim 241.9{ }^{\circ} \mathrm{C} ;{ }^{1} \mathrm{H}$ NMR $\left(\mathrm{D}_{2} \mathrm{O}, 400 \mathrm{MHz}\right) \delta$ : 9.30 (d, $J=2.0 \mathrm{~Hz}, 2 \mathrm{H}, \mathrm{ArH}), 9.03$ (d, $J=2.0 \mathrm{~Hz}, 2 \mathrm{H}, \mathrm{ArH}$ ), $4.24\left(\mathrm{t}, J=6.8 \mathrm{~Hz}, 2 \mathrm{H}, \mathrm{CONCH}_{2}\right), 3.13 \sim 3.31(\mathrm{~m}, 10 \mathrm{H}$, $\left.\mathrm{NCH}_{2}\right), 2.10 \sim 2.26\left(\mathrm{~m}, 6 \mathrm{H}, \mathrm{CH}_{2}\right) ;{ }^{13} \mathrm{C}$ NMR $\left(\mathrm{D}_{2} \mathrm{O}\right) \delta$ : $162.97,140.94,131.74,131.53,130.51,126.92,123.97$, 45.56, 44.77, 44.09, 44.02, 38.21, 36.58, 24.28, 23.78, 22.75; ESI-MS $m / z: 459.2(\mathrm{M}+\mathrm{H}-3 \mathrm{HCl})^{+}$. Anal. calcd for $\mathrm{C}_{21} \mathrm{H}_{29} \mathrm{Cl}_{3} \mathrm{~N}_{6} \mathrm{O}_{6} \cdot 6.0 \mathrm{H}_{2} \mathrm{O}$ : C 37. 31, H 6.11, N 12.43; found $\mathrm{C} 37.00, \mathrm{H} 6.33, \mathrm{~N} 12.34$.

3,6-二硝基-2-\{4-[3-(3-氨丙氨基)丙氨基]丁基 $\}-1 H$ 苯并异喹啉-1,3(2H)-二酮三盐酸盐(5b): 产率 $58.69 \%$. m.p. $249.5 \sim 251.1{ }^{\circ} \mathrm{C} ;{ }^{1} \mathrm{H}$ NMR $\left(\mathrm{D}_{2} \mathrm{O}, 400 \mathrm{MHz}\right) \delta$ : 9.20 (d, $J=2.0 \mathrm{~Hz}, 2 \mathrm{H}, \mathrm{ArH}), 8.85$ (d, $J=2.0 \mathrm{~Hz}, 2 \mathrm{H}, \operatorname{ArH})$, $4.06\left(\mathrm{t}, J=6.4 \mathrm{~Hz}, 2 \mathrm{H}, \mathrm{CONCH}_{2}\right), 3.12 \sim 3.27(\mathrm{~m}, 10 \mathrm{H}$, $\left.\mathrm{NCH}_{2}\right), 2.09 \sim 2.24\left(\mathrm{~m}, 4 \mathrm{H}, \mathrm{CH}_{2}\right), 1.80 \sim 1.92(\mathrm{~m}, 4 \mathrm{H}$, $\left.\mathrm{CH}_{2}\right) ;{ }^{13} \mathrm{C}$ NMR $\left(\mathrm{D}_{2} \mathrm{O}\right) \delta: 162.54,146.87,130.50,131.31$, 131.40, 126.85, 123.96, 47.44, 44.78, 44.73, 44.51, 40.32, $36.60,24.25,23.79,23.26,22.77$; ESI-MS $m / z: 473.2$ $(\mathrm{M}+\mathrm{H}-3 \mathrm{HCl})^{+}$. Anal. calcd for $\mathrm{C}_{23} \mathrm{H}_{33} \mathrm{Cl}_{3} \mathrm{~N}_{6} \mathrm{O}_{6} \cdot 0.8 \mathrm{H}_{2} \mathrm{O}$ : C 44.37, H 5.38, N 14.11; found C 44.54, H 5.35, N 13.74.

3,6-二硝基-2-\{3-[4-(3-氨丙氨基)丁氨基]丙基\}- $1 H$ 苯并异喹啉-1,3(2H)-二酮三盐酸盐(5c): 产率 $50.42 \%$. m.p. $242.0 \sim 243.6{ }^{\circ} \mathrm{C} ;{ }^{1} \mathrm{H}$ NMR $\left(\mathrm{D}_{2} \mathrm{O}, 400 \mathrm{MHz}\right) \delta: 9.30$ (d, $J=2.0 \mathrm{~Hz}, 2 \mathrm{H}, \mathrm{ArH}), 9.11$ (d, $J=2.0 \mathrm{~Hz}, 2 \mathrm{H}, \mathrm{ArH})$, 4.29 (t, $\left.J=6.8 \mathrm{~Hz}, 2 \mathrm{H}, \mathrm{CONCH}_{2}\right), 3.17 \sim 3.32(\mathrm{~m}, 10 \mathrm{H}$, $\left.\mathrm{NCH}_{2}\right), 2.14 \sim 2.21\left(\mathrm{~m}, 4 \mathrm{H}, \mathrm{CH}_{2}\right), 1.90 \sim 1.92(\mathrm{~m}, 4 \mathrm{H}$, $\left.\mathrm{CH}_{2}\right) ;{ }^{13} \mathrm{C}$ NMR $\left(\mathrm{D}_{2} \mathrm{O}\right) \delta: 163.07,147.03,131.81,131.61$, 130.58, 126.89, 124.03, 47.12, 47.08, 45.42, 44.64, 38.00, 36.66, 24.31, 23.82, 22.89 (2C); ESI-MS $m / z$ : 473.2 (M+ $\mathrm{H}-3 \mathrm{HCl})^{+}$. Anal. calcd for $\mathrm{C}_{22} \mathrm{H}_{33} \mathrm{Cl}_{3} \mathrm{~N}_{6} \mathrm{O}_{6} \cdot 1.30 \mathrm{H}_{2} \mathrm{O}: \mathrm{C}$ 43.65, H 5.60, N 13.88; found C 43.90, H 5.75, N 13.54.

3,6-二硝基-2-\{4-[4-(3-氨丙氨基)丁氨基]丁基\}- $1 H$ 苯并异喹啉-1,3(2H)-二酮三盐酸盐(5d): 产率 $42.13 \%$. m.p. $250.1 \sim 251.9{ }^{\circ} \mathrm{C} ;{ }^{1} \mathrm{H}$ NMR $\left(\mathrm{D}_{2} \mathrm{O}, 400 \mathrm{MHz}\right) \delta$ : 9.25 (d, $J=2.0 \mathrm{~Hz}, 2 \mathrm{H}, \mathrm{ArH}), 8.89$ (d, $J=2.0 \mathrm{~Hz}, 2 \mathrm{H}, \mathrm{ArH})$, 4.10 (t, $\left.J=6.8 \mathrm{~Hz}, 2 \mathrm{H}, \mathrm{CONCH}_{2}\right), 3.15 \sim 3.25(\mathrm{~m}, 10 \mathrm{H}$,
$\left.\mathrm{NCH}_{2}\right), 2.14 \sim 2.18\left(\mathrm{~m}, 2 \mathrm{H}, \mathrm{CH}_{2}\right), 1.83 \sim 1.88(\mathrm{~m}, 8 \mathrm{H}$, $\left.\mathrm{CH}_{2}\right) ;{ }^{13} \mathrm{C}$ NMR $\left(\mathrm{D}_{2} \mathrm{O}\right) \delta: 162.54,146.89,131.52,131.33$, 130.42, 126.65, 123.99, 47.29, 47.14, 46.96, 44.63, 40.30, 36.65, 24.30, 23.82, 23.29, 22.90 (2C); ESI-MS $m / z$ : 487.2 $(\mathrm{M}+\mathrm{H}-3 \mathrm{HCl})^{+}$. Anal. calcd for $\mathrm{C}_{23} \mathrm{H}_{35} \mathrm{Cl}_{3} \mathrm{~N}_{6} \mathrm{O}_{6} \cdot 0.7 \mathrm{H}_{2} \mathrm{O}$ : C 45.40, H 5.70, N 13.81; found C 45.70, H 5.63, N 13.49.

3,6-二硝基-2-\{3-[4-(4-氨丁氨基)丁氨基]丙基 $\}-1 H$ 苯并异喹啉-1,3(2H)-二酮三盐酸盐(5e): 产率 $41.34 \%$. m.p. $234.1 \sim 236.0{ }^{\circ} \mathrm{C} ;{ }^{1} \mathrm{H}$ NMR $\left(\mathrm{D}_{2} \mathrm{O}, 400 \mathrm{MHz}\right) \delta: 9.30$ (d, $J=2.0 \mathrm{~Hz}, 2 \mathrm{H}, \mathrm{ArH}), 9.02(\mathrm{~d}, J=2.0 \mathrm{~Hz}, 2 \mathrm{H}, \mathrm{ArH})$, $4.26\left(\mathrm{t}, J=6.8 \mathrm{~Hz}, 2 \mathrm{H}, \mathrm{CONCH}_{2}\right), 3.09 \sim 3.31(\mathrm{~m}, 10 \mathrm{H}$, $\left.\mathrm{NCH}_{2}\right), 2.22 \sim 2.25\left(\mathrm{~m}, 2 \mathrm{H}, \mathrm{CH}_{2}\right), 1.82 \sim 1.90(\mathrm{~m}, 8 \mathrm{H}$, $\left.\mathrm{CH}_{2}\right) ;{ }^{13} \mathrm{C}$ NMR $\left(\mathrm{D}_{2} \mathrm{O}\right) \delta: 162.93,146.95,131.73,131.52$, 130.52, 126.90, 123.99, 47.01, 47.07, 46.97, 45.42, 38.90, $38.10,24.31,24.00,22.92,22.90,22.83$; ESI-MS $m / z$ : $487.2(\mathrm{M}+\mathrm{H}-3 \mathrm{HCl})^{+}$. Anal. calcd for $\mathrm{C}_{23} \mathrm{H}_{35} \mathrm{Cl}_{3} \mathrm{~N}_{6} \mathrm{O}_{6} \cdot$ $0.5 \mathrm{H}_{2} \mathrm{O}$ : C 45.53, H 5.68, N 13.85; found C 45.57, H 5.86, $\mathrm{N} 13.49$.

3,6-二硝基-2-\{4-[4-(4-氨丁氨基)丁氨基]丁基 $\} 1 H$ 苯并异喹啉-1,3-(2H)-二酮三盐酸盐(5f): 产率 42.62\%. m.p. $242.4 \sim 244.1{ }^{\circ} \mathrm{C} ;{ }^{1} \mathrm{H}$ NMR $\left(\mathrm{D}_{2} \mathrm{O}, 400 \mathrm{MHz}\right) \delta: 9.22$ (d, $J=2.0 \mathrm{~Hz}, 2 \mathrm{H}, \mathrm{ArH}), 8.87(\mathrm{~d}, J=2.0 \mathrm{~Hz}, 2 \mathrm{H}, \mathrm{ArH})$, $4.05\left(\mathrm{t}, J=7.2 \mathrm{~Hz}, 2 \mathrm{H}, \mathrm{CONCH}_{2}\right), 3.03 \sim 3.21(\mathrm{~m}, 10 \mathrm{H}$, $\left.\mathrm{NCH}_{2}\right), 1.77 \sim 1.83\left(\mathrm{~m}, 12 \mathrm{H}, \mathrm{CH}_{2}\right) ;{ }^{13} \mathrm{C}$ NMR $\left(\mathrm{D}_{2} \mathrm{O}\right) \delta$ : $162.50,146.81,131.45,131.26,130.35,126.58,123.92$, 47.19, 46.93, 46.90 (2C), 40.26, 38.78, 24.20, 23.91, 23.22, 22.85 (2C), 22.77; ESI-MS $m / z: 501.3(\mathrm{M}+\mathrm{H}-$ $3 \mathrm{HCl})^{+}$. Anal. calcd for $\mathrm{C}_{24} \mathrm{H}_{37} \mathrm{Cl}_{3} \mathrm{~N}_{6} \mathrm{O}_{6} \cdot 2.0 \mathrm{H}_{2} \mathrm{O}: \mathrm{C} 44.76$, H 5.79, N 13.05; found C 44.61, H 6.01, N 13.00 .

\subsection{4中间体 $7 \mathbf{a} \sim 7 \mathbf{c}$ 合成}

中间体 $7 \mathbf{a} \sim 7 \mathbf{c}$ 按参考文献[26]进行合成.

\section{2 .5 萘酰亚胺-二胺缀合物 9 合成}

将化合物 $80.88 \mathrm{~g}(10 \mathrm{mmol})$ 溶于 $50 \mathrm{~mL}$ 乙腈中, 加 无水碳酸钾 $2.07 \mathrm{~g}(15 \mathrm{mmol})$, 室温摚拌 $15 \mathrm{~min}$, 升温至 $45{ }^{\circ} \mathrm{C}$, 分 3 批加入 $5 \mathrm{mmol}$ 的化合物 $7 \mathbf{a} \sim 7 \mathbf{c}$, 控温 45 ${ }^{\circ} \mathrm{C}$, 反应 $10 \sim 12 \mathrm{~h}$. 回收乙腈, 剩余物用 $30 \mathrm{~mL}$ 氯仿溶 解, $30 \mathrm{~mL} \times 3 \mathrm{Na}_{2} \mathrm{CO}_{3} \quad 10 \%$ 的水溶液洗涤, 氯仿层用无 水硫酸钠干燥, 回收氯仿, 剩余物用硅胶柱分离提纯, 分别得淡黄色或无色油状化合物 $\mathbf{8}$. 将得到的油状化合 物 8 溶于适量乙醇中, 冷却至 $0{ }^{\circ} \mathrm{C}$, 分别滴加 $4 \mathrm{~mol} / \mathrm{L}$ 盐酸的乙醇溶液适量, 自然升至室温, 搅拌过夜至有大 量固体生成, 过滤, 用重蒸无水乙醇洗涤 3 次, 干燥得 白色固体化合物 $9 \mathbf{a} \sim 9 \mathbf{c}^{[9]}$.

2-\{3-[-2 (二甲氨基乙氨基)丙基]- $1 H$-苯并 $\}$ 异喹啉1,3(2H)-二酮二盐酸盐(9a): 产率 $52.36 \%$. m.p. 270.9 
$272.3{ }^{\circ} \mathrm{C} ;{ }^{1} \mathrm{H}$ NMR $\left(\mathrm{D}_{2} \mathrm{O}, 400 \mathrm{MHz}\right) \delta: 7.52(\mathrm{~d}, J=8.0 \mathrm{~Hz}$, $2 \mathrm{H}, \operatorname{ArH}), 7.48(\mathrm{~d}, J=7.2 \mathrm{~Hz}, 2 \mathrm{H}, \mathrm{ArH}), 7.11$ (t, $J=7.6$ $\mathrm{Hz}, 2 \mathrm{H}, \mathrm{ArH}), 3.53 \sim 3.63\left(\mathrm{~m}, 6 \mathrm{H}, \mathrm{CONCH}_{2}, 2 \times \mathrm{NCH}_{3}\right)$, $3.14\left(\mathrm{t}, J=7.2 \mathrm{~Hz}, 2 \mathrm{H}, \mathrm{NCH}_{2}\right), 2.98\left(\mathrm{~s}, 6 \mathrm{H}, 2 \times \mathrm{CH}_{3}\right)$, $1.82 \sim 1.90\left(\mathrm{~m}, 2 \mathrm{H}, \mathrm{CH}_{2}\right) ;{ }^{13} \mathrm{C}$ NMR $\left(\mathrm{D}_{2} \mathrm{O}\right) \delta: 161.94$, $132.71,128.70,127.67,124.46,123.32,116.94,50.22$, 43.79, 41.22 (2C), 39.65, 34.94, 21.96; ESI-MS $m / z: 326.2$ $(\mathrm{M}+\mathrm{H}-2 \mathrm{HCl})^{+}$. Anal. calcd for $\mathrm{C}_{19} \mathrm{H}_{25} \mathrm{Cl}_{2} \mathrm{~N}_{3} \mathrm{O}_{2} \bullet 0.5 \mathrm{H}_{2} \mathrm{O}$ : C 56.02, H 6.43, N 10.32; found C 55.83, H 6.12, N 10.23.

2-\{4-[-2 (二甲氨基乙氨基)丁基] $1 H$-苯并\}异喹啉1,3(2H)-二酮二盐酸盐(9b): 产率 46.17\%. m.p. 246.6 $248.0{ }^{\circ} \mathrm{C} ;{ }^{1} \mathrm{H}$ NMR $\left(\mathrm{D}_{2} \mathrm{O}, 400 \mathrm{MHz}\right) \delta: 8.11(\mathrm{~d}, J=6.0 \mathrm{~Hz}$, 2H, ArH), 8.09 (d, $J=6.0 \mathrm{~Hz}, 2 \mathrm{H}, \operatorname{ArH}), 7.01$ (t, $J=8.0$ $\mathrm{Hz}, 2 \mathrm{H}, \mathrm{ArH}), 3.92$ (t, $J=6.4 \mathrm{~Hz}, 2 \mathrm{H}, \mathrm{CONCH}_{2}$ ), 3.55 $3.58\left(\mathrm{~m}, 4 \mathrm{H}, \mathrm{NCH}_{2}\right), 3.26\left(\mathrm{t}, J=7.2 \mathrm{~Hz}, 2 \mathrm{H}, \mathrm{NCH}_{2}\right), 3.02$ (s, $\left.6 \mathrm{H}, 2 \times \mathrm{CH}_{3}\right), 2.07 \sim 2.15\left(\mathrm{~m}, 4 \mathrm{H}, \mathrm{CH}_{2}\right) ;{ }^{13} \mathrm{C} \mathrm{NMR}$ $\left(\mathrm{D}_{2} \mathrm{O}\right) \delta: 164.43,135.16,131.43,130.93,127.36,126.74$, 120.71, 52.03, 47.88, 43.51 (2C), 41.81, 39.60, 24.05, 23.55; ESI-MS $m / z: 340.3(\mathrm{M}+\mathrm{H}-2 \mathrm{HCl})^{+}$. Anal. calcd for $\mathrm{C}_{20} \mathrm{H}_{27} \mathrm{Cl}_{2} \mathrm{~N}_{3} \mathrm{O}_{2} \bullet 0.15 \mathrm{H}_{2} \mathrm{O}$ : C 57.88, $\mathrm{H}$ 6.63, N 10.12; found $\mathrm{C} 57.48, \mathrm{H} 6.28, \mathrm{~N} 9.78$.

2-\{5-[-2 (二甲氨基乙氨基)戊基]- $1 H$-苯并 $\}$ 异喹啉1,3(2H)-二酮二盐酸盐(9c): 产率 45.21\%. m.p. 259.1 $260.6{ }^{\circ} \mathrm{C} ;{ }^{1} \mathrm{H}$ NMR $\left(\mathrm{D}_{2} \mathrm{O}, 400 \mathrm{MHz}\right) \delta: 7.96(\mathrm{~d}, J=8.0 \mathrm{~Hz}$, $2 \mathrm{H}, \operatorname{ArH}), 7.50$ (d, $J=7.6 \mathrm{~Hz}, 2 \mathrm{H}, \operatorname{ArH}), 7.11$ (t, $J=7.2$ $\mathrm{Hz}, 2 \mathrm{H}, \mathrm{ArH}), 3.81$ (t, $\left.J=6.8 \mathrm{~Hz}, 2 \mathrm{H}, \mathrm{CONCH}_{2}\right), 3.64 \sim$ $3.69\left(\mathrm{~m}, 4 \mathrm{H}, \mathrm{NCH}_{2}\right), 3.26\left(\mathrm{t}, J=7.2 \mathrm{~Hz}, 2 \mathrm{H}, \mathrm{NCH}_{2}\right), 3.12$ (s, $\left.6 \mathrm{H}, 2 \times \mathrm{CH}_{3}\right), 1.86 \sim 1.92\left(\mathrm{~m}, 2 \mathrm{H}, \mathrm{CH}_{2}\right), 1.54 \sim 1.63(\mathrm{~m}$, $\left.2 \mathrm{H}, \mathrm{CH}_{2}\right), 1.47 \sim 1.52\left(\mathrm{~m}, 2 \mathrm{H}, \mathrm{CH}_{2}\right) ;{ }^{13} \mathrm{C}$ NMR $\left(\mathrm{D}_{2} \mathrm{O}\right) \delta$ : $166.11,136.93,133.20,132.83129 .27,128.60,122.70$, $54.69,50.13,45.54$ (2C), 43.80, 41.97, 29.14, 27.58, 25.67; ESI-MS $m / z: 354.3(\mathrm{M}+\mathrm{H}-2 \mathrm{HCl})^{+}$. Anal. calcd for $\mathrm{C}_{21} \mathrm{H}_{29} \mathrm{Cl}_{2} \mathrm{~N}_{3} \mathrm{O}_{2} \bullet 1.2 \mathrm{H}_{2} \mathrm{O}$ : C 56.30, $\mathrm{H}$ 7.06, N 9.38; found $\mathrm{C} 56.02, \mathrm{H} 6.71, \mathrm{~N} 9.30$.

\section{3 体外抗肿瘤活性}

为了考察本系列化合物抗肿瘤活性, 采用 MTT [3-(4,5-二甲基噻唑-2)-2,5-二苯基四氮唑溴盐]法 ${ }^{[15]}$ 进行 初步篎选, 对照品为 Amonafide.

细胞培养条件: 取繁殖期细胞, 加入含 $10 \%$ 小牛血 清, $2 \mathrm{mmol} / \mathrm{L} L$-谷氨酸, $100 \mathrm{U} / \mathrm{mL}$ 青霉素, $50 \mu \mathrm{g} / \mathrm{mL}$ 链 霰素、 $2 \mathrm{mmol} / \mathrm{L}$ 氨基胍培养液中, 于 $37{ }^{\circ} \mathrm{C}, 5 \% \mathrm{CO}_{2}$ 环 境中睬育.

细胞毒性测试: 分别取对数生长期的人乳腺癌细胞 (MB-231) 和肝癌细胞 (7721), 调整细胞数为 $5 \times 10^{3}$ 个 $/ \mathrm{mL}$, 加于 96 孔培养板中使其贴壁过夜, $24 \mathrm{~h}$ 后加入已
知浓度的样品液, 再过 $48 \mathrm{~h}$ 加 MTT 溶液, 每孔 $100 \mu \mathrm{L}$; 在 $37{ }^{\circ} \mathrm{C}$ 条件下于培养箱中孵育 $4 \mathrm{~h}$, 除去 MTT 溶液, 将每孔细胞晶体用 $150 \mu \mathrm{L}$ DMSO 溶解. K562(白血病细 胞) $4 \times 10^{3}$ 个 $/ \mathrm{mL}$ 加于 96 孔培养板中, 加入已知浓度的 样品液, 再过 $48 \mathrm{~h}$ 加 MTT 溶液, 每孔 $10 \mu \mathrm{L}(10$ $\mathrm{mg} / \mathrm{mL}$ ); 在 $37{ }^{\circ} \mathrm{C}$ 条件下于培养箱中孵育 $4 \mathrm{~h}$, 除去 MTT 溶液, 将每孔细胞晶体用 $10 \%$ 的 SDS(十二烷基硫 酸钠)溶液 $100 \mu \mathrm{L}$ 溶解过夜. 以上均分为样品组、对照 组(不加样品)和空白组(只有培养基, 无细胞), 在酶标 仪上测定波长 $570 \mathrm{~nm}$ 处得的光密度值, 然后由下面的 公式计算不同样品的抑制率:

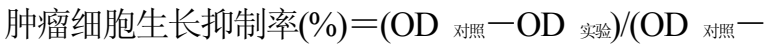
$\mathrm{OD}$ 空白 $) \times 100 \%$

\section{4 荧光光谱学研究}

Tris- $\mathrm{HCl}$ 缓冲溶液配制: 称取三羟甲基氨基甲烷 (Tris) $12.11 \mathrm{~g}$, 用蒸馏水稀释至 800 900 mL, 用 $20 \%$ 的 盐酸水溶液调节 $\mathrm{pH}=7.4$, 配成 $\mathrm{Tris}-\mathrm{HCl}$ 缓冲液 1000 $\mathrm{mL}$.

化合物 5a 溶液配制: 称取化合物适量, 用 Tris-HCl 缓冲液配成浓度为 $2.0 \times 10^{-4} \mathrm{~mol} / \mathrm{L}$ 的溶液 $50 \mathrm{~mL}$.

鲱鱼精 DNA 溶液配制: 称取鲱鱼精 DNA $15.8 \mathrm{mg}$ 用 Tris- $\mathrm{HCl}$ 缓冲液配制成浓度为 $22.82 \times 10^{-5} \mathrm{~mol} \cdot \mathrm{L}^{-1}$ 的溶液 $50 \mathrm{~mL}$.

溴化乙锭溶液配制: 称取澳化乙锭 $(\mathrm{EB}) 3 \mathrm{mg}$, 用 Tris- $\mathrm{HCl}$ 缓冲液配制成浓度为 $1.57 \times 10^{-5} \mathrm{~mol} \cdot \mathrm{L}^{-1}$ 的溶 液 $50 \mathrm{~mL}$.

鲱鱼精 DNA $0.3 \mathrm{~mL}$, EB 缓冲溶液 $0.5 \mathrm{~mL}$ 分别与 $0.0,0.1,0.2,0.3,0.6,0.9,1.2,1.5,1.8,2.1,2.7,3.0 \mathrm{~mL}$ 化 合物 5a 溶液混合, 然后分别用 Tris- $\mathrm{HCl}$ 缓冲溶液稀释 至 $5 \mathrm{~mL}$, 摇匀、静置 $0.5 \mathrm{~h}$ 进行荧光测定, 测定时温度 分别为 $25,30,37{ }^{\circ} \mathrm{C}$. 样品中 $\mathbf{5 a}$ 浓度分别为: $0.0,4 \times$ $10^{-6}, 8 \times 10^{-6}, 12 \times 10^{-6}, 24 \times 10^{-6}, 36 \times 10^{-6}, 48 \times 10^{-6}$, $60 \times 10^{-6}, 72 \times 10^{-6}, 84 \times 10^{-6}, 96 \times 10^{-6}, 108 \times 10^{-6}$, $120 \times 10^{-6} \mathrm{~mol} \cdot \mathrm{L}^{-1}, \mathrm{DNA}$ 和 $\mathrm{EB}$ 浓度分别为 $13.7 \times 10^{-6}$ 和 $15.7 \times 10^{-6} \mathrm{~mol} \cdot \mathrm{L}^{-1}$.

经在 $25{ }^{\circ} \mathrm{C}$ 测定, 化合物 $\mathbf{5 a}$ 在水中溶解度大于 80 $\mathrm{mg} / \mathrm{mL}$ ，但据文献 ${ }^{[27,28]}$ 报道 Amonafide 难溶于水.

\section{References}

[1] Braña, M. F.; Romas, A. Curr. Med. Chem.: Anti-Cancer Agents 2001, 1, 237.

[2] Ingrassia, L.; Lefranc, F.; Kiss, R.; Mijatovic, T. Curr. Med. Chem. 2009, 16, 1192.

[3] Wang, Y.-X.; Zhang, X.-B.; Zhao, J.; Xie, S.-Q.; Wang, C.-J. J. Med. Chem. 2012, 16, 3502.

[4] Braña, M. F.; Beatriz-Sáez, G. D.; Simmon-Robinson, C. R.; Barlozzarib, T. Eur. J. Med. Chem. 2002, 37, 541. 
[5] Papadopoulou. M. V; Rosenzweig, H. S.; Bloomer, W. D. Bioorg. Med. Chem. Lett. 2004, 14, 1519.

[6] Burns, M. R.; Wood, S. J.; Miller, K. A.; Nguyen, T.; Cromerb, J. R.; David, S. A. Bioorg. Med. Chem. 2005, 13, 2523.

[7] Wolf, M.; Bauder-Wust, U.; Pipkorn, R.; Eskerskic, H.; Eisenhuta, M. Bioorg. Med. Chem. Lett. 2006, 16, 3193.

[8] Moret, V.; Dereudre-Bosquet, N.; Clayette, P.; Laras, Y.; Pietrancosta, N.; Rolland, A.; Weck, C.; Marc, S.; Kraus, J.-L. Bioorg. Med. Chem. Lett. 2006, 16, 5988.

[9] Tian, Z.-Y.; Xie, S.-Q.; Du, Y.-W.; Ma, Y.-F.; Zhao, J.; Gao, W.-Y.; Wang, C.-J. Eur. J. Med. Chem. 2009, 44, 393.

[10] Tian, Z.-Y.; Ma, H.-X.; Xie, S.-Q.; Wang, X.; Zhao, J.; Wang, C.-J.; Gao. W.-Y. Chin. Chem. Lett. 2008, 19, 509.

[11] Tian, Z.-Y.; Xie, S.-Q.; Mei, Z.-H.; Zhao, J.; Gao, W.-Y.; Wang, C.-J. Org. Biomol. Chem. 2009, 7, 4551.

[12] Mei, Z.-H.; Tian, Z.-H.; Ma, H.-X.; Xie, S.-Q.; Zhao, J.; Wang, C.-J. Acta Pharm. Sin. 2009, 44, 754 (in Chinese). (梅子厚, 田智勇, 马红霞, 谢松强, 赵瑾, 王超杰, 药学学报, 2009, 44, 754.)

[13] Tian, Z.-Y.; Su, L.-P.; Wang, D.; Li, Y.-M.; Zhao, J.; Wang, C.-J. Chem. Res. 2012, 23, 24 (in Chinese).

(田智勇, 苏雷朋, 王丹, 李亚明, 赵瑾, 王超杰, 化学研究, 2012, 23, 24.)

[14] Zee, C. R. K. Y; Cheng, C. C. J. Med. Chem., 1985, 28, 1216.

[15] Mosmann, T. J. Immunol. Methods 1983, 65, 55.

[16] Li, F.-M. Textbook of Analytical Chemistry, People's Medical Publishing House, Beijing, 2011, pp. 217 218 (in Chinese). (李发美, 分析化学, 人民卫生出版社, 北京, 2011, pp. 217 218.)

[17] Lv, J.; Wang, G.-K., Zhang, G.-S.; Liu, Q.-F.; Lu, Y. Spectrosc. Spectr. Anal. 2010, 30, 1324 (in Chinese).
(吕娟, 王公辑, 张贵生, 刘青锋, 卢膺, 光谱学与光谱分析, 2010, 30, 1324.)

[18] Yu, L.-L.; Yang, R.; Bai, X.-X.; Chen, X.-Y.; Li, J.-J.; Qu, L.-B. Chin. J. Lumin. 2011, 32, 1997 (in Chinese).

(于岗岗, 杨舟, 白希希, 陈晓英, 李建军, 屈凌波, 发光学报, 2011, 32, 1997.)

[19] Yan, C.-N.; Pan, Z.-T.; Liu, Y.; Qu, S.-S. Chin. J. Anal. Chem. 2004, 32, 317 (in Chinese).

(颜承农, 潘祖亭, 刘义, 屈松生, 分析化学, 2004, 32, 317.)

[20] Ashoka, S.; Seetharamappa, J.; Kandagal, P. B.; Shaikh, S. M. T. J. Lumin. 2006, 121, 179.

[21] Liu, B.-S.; Yang, C.; Wang, J.; Xie, C.-L.; Lv, Y.-K. Chin. J. Lumin. 2011, 32, 293 (in Chinese).

(刘保生, 杨超, 王晶, 薛春丽, 吕运开, 发光学报, 2011, 32, 293.)

[22] Klotz, I. M.; Urquhart, J. M. J. Am. Chem. Soc. 1949, 71, 847.

[23] Bi, S.-Y; Qiao, C.-Y.; Song, D.-Y.; Tian, Y.; Gao, D.-J.; Zhang, H. Q. Sens. Actuators, B 2006, 119, 199.

[24] Takenaka, S.; Ihara, T.; Takagi, M. J. J. Chem. Soc., Chem. Commun. 1990, 21, 1485.

[25] Tian, Z.-Y.; Xie, S.-Q.; Zhao, J.; Gao, W.-Y.; Wang, C.-J. Chem. Res. 2009, 30, 33 (in Chinese).

(田智勇, 魏金风, 谢松强, 赵瑾, 高文远, 王超杰, 化学研究, 2009, 30, 33.)

[26] Kamal, A.; Reddy, B. S. N.; Reddy, G. S. K.; Ramesh, G. Bioorg. Med. Chem. Lett. 2002, 12, 1933.

[27] Barron, G. A.; Bermano, G.; Gordon, A.; Thoo Lin, P. K. Eur. J. Med. Chem. 2010, 45, 1430.

[28] Braña, M. F.; Castellano, J. M.; Morán, M.; Pérez de Vega, M. J.; Qian, X.-D.; Romerdahl, C. A.; Keilhauer, G. Eur. J. Med. Chem. 1995, 30, 235.

(Qin, X.) 